\title{
Geomagnetic reversals at the edge of regularity
}

\author{
Breno Raphaldini, ${ }^{1}$ Everton S. Medeiros, ${ }^{2}$ David Ciro $\odot,{ }^{3}$ Daniel Ribeiro Franco $\odot,{ }^{4}$ and Ricardo I. F. Trindade $\odot^{5}$ \\ ${ }^{1}$ Department of Mathematical Sciences, Durham University, Upper Mountjoy Campus, Stockton Road, Durham DH1 3LE, United Kingdom \\ ${ }^{2}$ Institut für Theoretische Physik, Technische Universität Berlin, Hardenbergstraße 36, 10623 Berlin, Germany \\ ${ }^{3}$ Centro de Ciências Biológicas, Universidade Federal de Santa Catarina, Florianópolis, Brazil \\ ${ }^{4}$ Observatorio Nacional, 04533-085, Rio de Janeiro, Brazil \\ ${ }^{5}$ Instituto de Astronomia Geofísica e Ciências Atmosféricas - Universidade de São Paulo, 05508-090 São Paulo, Brazil
}

(Received 16 June 2020; accepted 1 February 2021; published 18 February 2021)

\begin{abstract}
Geomagnetic field reversals remain as one of the most intriguing problems in geophysics and are regarded as chaotic processes resulting from a dynamo mechanism. In this article we use the polarity scale data of the last 170 Myrs collected from the ocean floor to provide robust evidence for an inverse relationship between the complexity of sequences of consecutive polarity intervals and the respective reversal rate. In particular, the variability of sequences of polarity intervals reaches minimum values in the mid-Jurassic when a maximum reversal rate is found, in the early Cretaceous preceding the Cretaceous Superchron, and twice in the last 20 Myrs. These facts raise the possibility of epochs of high regularity in the geomagnetic field reversals. To shed light on this process, we investigate the transition from regular to chaotic regime in a minimal model for geomagnetic reversals. We show that even in chaotic regimes, the system retains the signature of regular behavior near to transitions. We suggest that geomagnetic reversals have switched between different degrees of irregularity, with a dominant periodicity of $\approx 70 \mathrm{kyrs}$ that results from the occurrence of "ghost" limit cycles (GLCs) or unstable periodic orbits (UPOs) immersed in a chaotic region of phase space.
\end{abstract}

DOI: 10.1103/PhysRevResearch.3.013158

\section{INTRODUCTION}

The geomagnetic field is characterized by a dominant dipole component that reverses its polarity in irregular times. The physical mechanism driving reversals of the geomagnetic field's dipole is still not well understood, although it is well established that reversals are linked to the dynamo process that takes place on Earth's outer core [1]. It is hypothesized that long term changes in the reversal processes must be linked to the evolution of the dynamo process in the Earth's outer core. For instance the growth of the inner core [2], and most notably the changes in the properties of the core-mantle boundary, could have large impacts in the reversal process $[3,4]$.

Polarity scales compiled from paleomagnetic data reveal a large variability of the polarity intervals $[5,6]$. Short intervals have a duration of an order of tens of thousands of years, while exceptionally long intervals with a single polarity last $O\left(10^{7}\right)$ yrs and are called superchrons. These events have occurred at least three times in the palemagnetic record. The first superchron is the Cretaceous Superchron, roughly from about 120 to 80 million years ago. The second one is named Kiaman Superchron and occurred in the Carboniferous-Permian, roughly between 320 and 270 million years ago. The third one is called Moyero Superchron [7], and occurred in the Ordovician being

Published by the American Physical Society under the terms of the Creative Commons Attribution 4.0 International license. Further distribution of this work must maintain attribution to the author(s) and the published article's title, journal citation, and DOI. the shortest of the three, lasting less than 20 million years (485 to 463 million years ago). On the other hand, an epoch of exceptionally fast reversal rates occurred in the mid-Jurassic (155-170 million years ago), with an reversal rate of over 10 reversals per million years.

The statistics and variability of reversal rates is a matter of debate. It was long assumed the reversal rates to follow a Poisson type of renewal process. This leads to an exponential distribution $p(T) \propto \exp (-\lambda T)$, where $\lambda$ is the rate of the process. This type of process is memoryless leading to an independent sequence of polarity intervals, see $[8,9]$. Subsequent articles investigated the hypothesis of a Poisson process in which the rate itself evolves in time $\lambda=\lambda(t)$ [10]. Carbone et al. [11], later on, showed that the sequences of reversals largely departs from a Poisson process. They suggested that reversals are better modeled by a Levy-type process. This result contrasts from previous research, implying in long-range correlations between intervals duration, with different classes of intervals clustered in time. However, the physical mechanism behind these long-range dependencies remains elusive. Insights are provided from other natural dynamos, such as the Sun and other stars usually operating in a quasiperiodic cyclic manner. The Sun has a well-recorded 11 year cycle, named Schwabe cycle, with peaks in sunspot numbers each 11 years on average occurring in antiphase with its axial dipole dynamics [12]. Despite possessing a strong regular component the solar cycle also displays irregular behavior such as the Maunder Minimum, which was suggested to arise from intermittent type of behavior [13,14]. See [15] for a comprehensive review. This analogy raise the question of whether the geodynamo operates in more regular regimes similar to other 
natural dynamos, and if so, what type of signature this regime on the edge between regular and chaotic would leave on the observational record.

In this work we address this issue by searching for regularities in the sequence of geomagnetic reversals recorded in the last 170 Myrs using data from a well known geomagnetic polarity scale [6]. Specifically, we assess the level of regularity in this signal by estimating its sample entropy (SamEn) and coefficient of variation $(C)$. With this we provide statistical evidence for significant variations in the signal's regularity at the timescale of $10^{7} \mathrm{yrs}$. And, more importantly, we find several periods of highly regular reversals. First, and most noticeable, in the mid-Jurassic accompanied by an extreme reversal rate, other in the early Cretaceous, preceding the Cretaceous Normal Superchron, and twice in the last 20 Myrs. All these periods correspond to a high reversal rate. The overall irregularity of the signal is evidenced in the large variability in the density polarity intervals, however, we observe preferential intervals pointing out to underlying periodic processes. Finally, we interpret these results in the framework of nonlinear dynamics by analyzing the transitions from regular to chaotic behavior in a simple model for geomagnetic reversals.

\section{MEASURES OF REGULARITY}

To quantify the degree of regularity in the geomagnetic reversals we invoke the concept of chaos from nonlinear dynamics. Chaos is usually characterized by the exponential separation of nearby trajectories in the system's state space as the time advances [16]. The rate of such separation is quantified by the Lyapunov spectrum $\lambda_{i}(x)$ [17]. Another way of measuring the complexity of a dynamical system is the Kolmogorov-Sinai (KS) entropy that qualitatively measures the rate of creation of information of the system [17,18]. For a class of dynamical systems [16], the KS entropy and the Lyapunov spectrum are linked by the Pesin identity:

$$
\mathcal{S}_{\mathrm{KS}}=\int \sum_{\lambda>0} \lambda_{i}(x) d \chi
$$

where $\chi$ is the ergodic invariant probability measure of the chaotic attractor. The KS entropy is therefore the sum of all positive Lyapunov exponents of the system.

Sample entropy: For applications to real-world data, definition (1) needs to be adapted to overpass intrinsic difficulties arising from observational procedures. For this task, a measure called approximate entropy (ApEn) [19] and its successor sample entropy (SamEn) [20] have been successfully employed to quantify the amount of regularity in electrocardiograms [21], and cardiovascular signals in general [22]. A comprehensive review of ApEn and SamEn is found in [23]. Here we implement SamEn to assess the level of regularities in the dynamics of reversals of the geomagnetic field. Given a data sample $X=\left(x_{1}, \ldots, x_{N}\right)$, a subset of $X$ of size $m$ is written as $X_{m}(i)=\left(x_{i}, \ldots, x_{i+m-1}\right)$ with $1 \leqslant i \leqslant i+m+$ $1 \leqslant N$. To calculate SamEn define $A=$ number of times vectors with size $m+1$ such that $d\left(X_{m+1}(j), X_{m+1}(i)\right)<r$, and define $B=$ number of times vectors with size $m$ such that $d\left(X_{m}(j), X_{m}(i)\right)<r$, where $d(.,$.$) is any suitable distance$ (here we use the Euclidean distance). Now define

$$
\operatorname{SamEn}(m, R, N)=-\log \left(\frac{A}{B}\right)=-\log \left[\frac{\sum_{i=1}^{N-m} \sum_{j=1, j \neq 1}^{N-m} \#\left[d\left(x_{m+1}(j), x_{m+1}(i)\right)<r\right]}{\sum_{i=1}^{N-m} \sum_{j=1, j \neq 1}^{N-m} \#\left[d\left(x_{m}(j), x_{m}(i)\right)\right]<r}\right] .
$$

where \# denotes the number of elements in a set. In our analysis we adopt commonly used values of $r$ and $m$, namely, $m=2$ and $r=0.2 \sigma(X)$ [20], where $\sigma(X)$ denotes the standard deviation of the sample $X$, the results remain valid with slight change in the used parameters (sensibility test using different $m$ and $r$ parameters is provided in the Supplemental Material [24]).

Coefficient of variation: We complement our analysis on the dispersion of the persistence times by calculating the coefficient of variation $C=\sigma(X) / \mathbb{E}(X)$, where $E$ is the expected value of the random variable $X$. The coefficient of variation has an important implication for our analysis, since for any random variable $X$ with an exponential distribution $C(X)=1$. Indeed, for a random variable $X$ with exponential distribution $p(X)=\lambda \exp (-\lambda t)$ we have $\mathbb{E}(X)=1 / \lambda$ and $\sigma=\sqrt{\operatorname{Var}(X)}=1 / \lambda$, therefore, $\sigma / \mathbb{E}(X)=1 . C(X) \neq 1$ is implied in a departure from the Poissonian property. Therefore the coefficient of variation is a tool that enables us to evaluate the Poissonian hypothesis $[9,10]$.

\section{DATA AND OBSERVATIONAL RESULTS}

Here we use the geomagnetic polarity timescale compiled by Gradstein et al. [6]. Polarity timescales often rely on measurements from different natures, for instance magnetostratigraphy, biostratigraphy, and radio isotope dating $[25,26]$. Most precise knowledge on geomagnetic reversal sequences is obtained from the available data of seafloor magnetization. As the magma cools on spreading ridges, minerals containing iron and other magnetic materials record the external magnetic field. The geomagnetic polarity timescale provided by Gradstein et al. [6] is restricted to the period when seafloor data is available, namely, starting in the midJurassic, comprising the $C$ sequence (83 Myr-present) and the $M$ sequence (170-83 Myrs). The geomagnetic polarity timescale used here is presented in Fig. 1. The determination of the duration of a polarity interval is constrained by models of the seafloor spreading rate and complemented

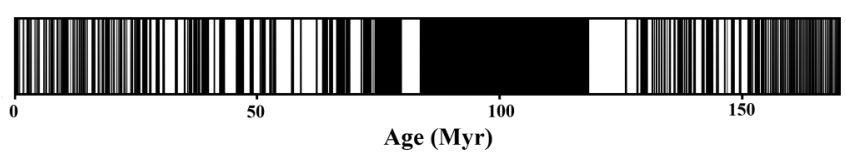

FIG. 1. Geomagnetic polarity timescale presented by Gradstein et al. [6], times with normal polarity are marked in black, while times with reverse polarity are marked in white. 
by magnetostratigraphic data. Polarity information from the mid-Jurassic is constrained by the Japanese lineations in the Pacific plate. Such lineations have a faster spreading rate [27] compared to the slow formation of the Hawaiian lineations, used in previous polarity scales, such as [5]. Concerning the reliability of the analyzed records, we observe that in a study for the mid-Jurassic data from the Pacific plate [27], point out to a particular period with problematic data (from 162 to 167 Myrs) for which the records are poorly constrained. However, for 155-162 Myrs, the same authors have reported robust results for the polarity intervals. Besides, for this period, Gradstein et al. [6] use several sources of magnetostratigraphic sequences to constrain the polarity scale. Finally, most reliable data, i.e., polarity intervals recorded in more than one plate, are available already from 154.3 Myrs ago (M25 Chron) $[6,28]$.

With this, in Figs. 2(a) and 2(b) we show the coefficient of variation $C$ and SamEn, respectively. These measures are evaluated from the geomagnetic reversals data for sequences of 30 consecutive polarity intervals of duration $\Delta T$. These intervals are shown Figs. 2(a) and 2(b) as $-\log (\Delta T)$ (black curve is not defined during the superchron when the reversal rate approaches 0 ) for scaling purposes. There is an apparent inverse relationship between the regularity of the sequences of intervals and the corresponding reversal rate, with a particularly ordered behavior in the mid-Jurassic $(\approx 152-165$ Myrs ago). In Fig. 2(c) we show the distribution of intervals with sizes restricted to $\Delta T \leqslant 600$ kyrs. This histogram reveals a clustered character in the distribution of intervals with several peaks of occurrences, and the most frequent being $\Delta t \approx 70$ kyrs. A possible interpretation for these peaks is the occurrence of different dynamical regimes of the geodynamo. Such variability of regimes could be a result of changes in the core-mantle boundary [29], or even a stochastic resonance [30] at which regularity is induced by the interaction of noise and external periodic forcing.

Moreover, the most frequent interval being $\Delta T \approx 70 \mathrm{kyrs}$ suggests that fast-reversing regimes are of high relevance for the geodynamo. This observation is compatible with the range of intervals found at around 155-170 Myrs ago that are seemingly regular in accordance with Figs. 2(a) and 2(b). More recently, Gallet et al. [31] reported a period with similar reversal rates at the Drumian (around 504.5-500.5 Myrs ago). Another interesting outcome of the analysis presented in Fig. 2(a) is that $C$ can be viewed as a measure of the Poissonity of the distribution of reversal times, with $C \neq 1$ compatible with a large departure from Poissonity. This is the case in sequences in the vicinity of the superchron nonreversing state $(\approx 80-120$ Myrs $)$ and in regular state near $\approx 160$ Myrs. An important implication of this observation is the validity of models that describe the geomagnetic reversals as a stochastic exit problem with Poisson times [32,33], in this case $C$ could be used to assess the periods in the past when these models are compatible with the observations.

Since the determination of the duration of the polarity intervals is subject to errors, which is especially relevant for the pre-154.3 Myr period, it is important to test how the coefficient of variation $(C)$ and the sample entropy (SamEn) behave when the data are subject to random perturbations. In order to test the robustness of our results, we introduce random errors
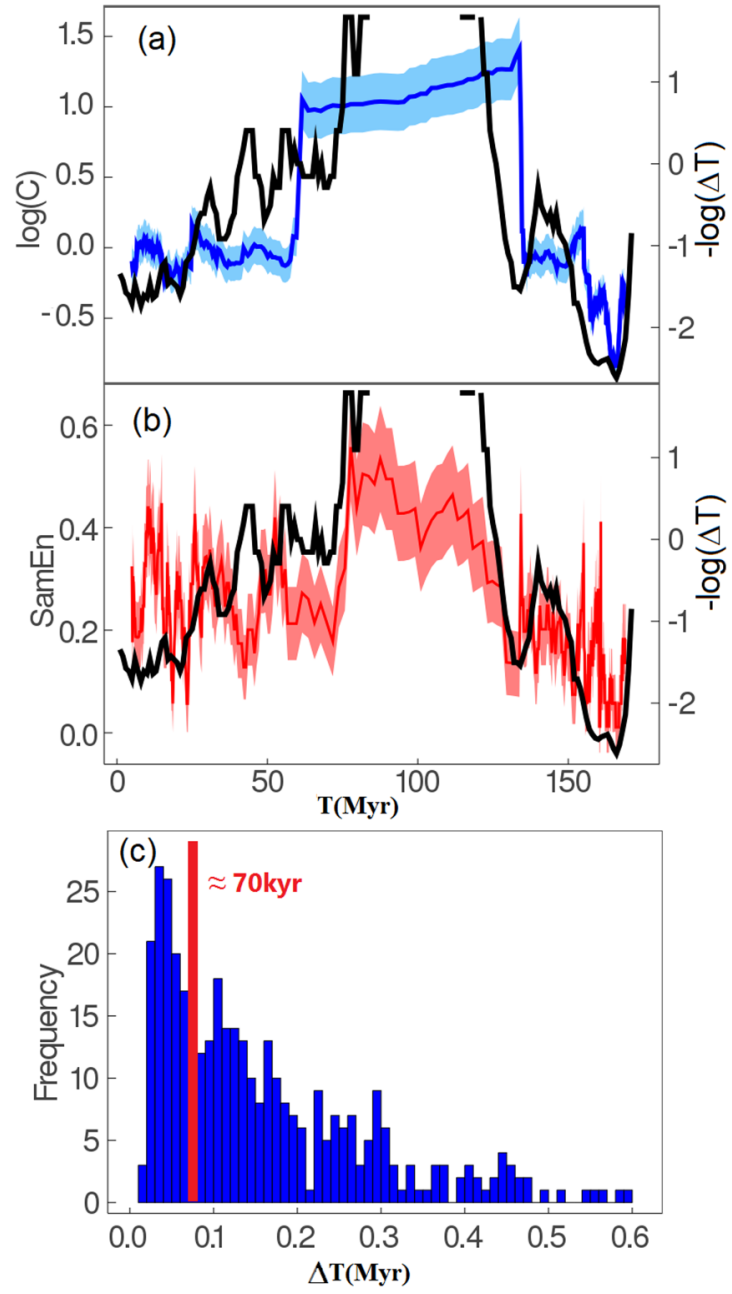

FIG. 2. (a) The blue curve represents coefficient of variation $(C)$ calculated for the geomagnetic polarity scale. (b) The red curve stands for the sample entropy (SamEn) calculated for the geomagnetic polarity scale using parameters $r=0.2 \sigma(X)$ and $m=2$. The black curve in (a) and (b) represents $-\log (\Delta T)$ where $\Delta T$ corresponds to polarity intervals. Confidence intervals with $95 \%$ confidence levels are calculated by bootstrap resampling. (c) Histogram of polarity intervals for $T \leqslant 600 \mathrm{kyrs}$ with the dominant $\approx 70 \mathrm{kyrs}$ bin highlighted in red.

to the polarity interval data set, following a few constraints: (a) when a given polarity interval is perturbed by the error, it must remain positive; and (b) the error should be larger for longer polarity intervals. With these constraints we proceed in the following way: let $X_{i}(i=1,2, \ldots, N)$ be the random variable representing the duration of the $i$ th polarity interval, let $\epsilon_{i}$ be a normal random variable with mean $\mathbb{E}(X)=-\sigma^{2} / 2$ and variance $\sigma^{2}$. The errors are then introduced multiplicatively as $\tilde{X}_{i}=\exp \left(\epsilon_{i}\right) X_{i}$. This corresponds to introducing multiplicative errors with lognormal distribution, the values of the mean and variance are chosen so that $\mathbb{E}\left[\exp \left(\epsilon_{i}\right)\right]=1$. We perform the calculations for different values of the variance, namely, $\sigma=0.1, \sigma=0.2$, and $\sigma=0.5$. We see in Fig. 3 that the time evolution of $C$ and SamEn are not significantly affected by including the errors, but there is a monotonic increase of the coefficient of variation as function of the level of noise. 
(a)
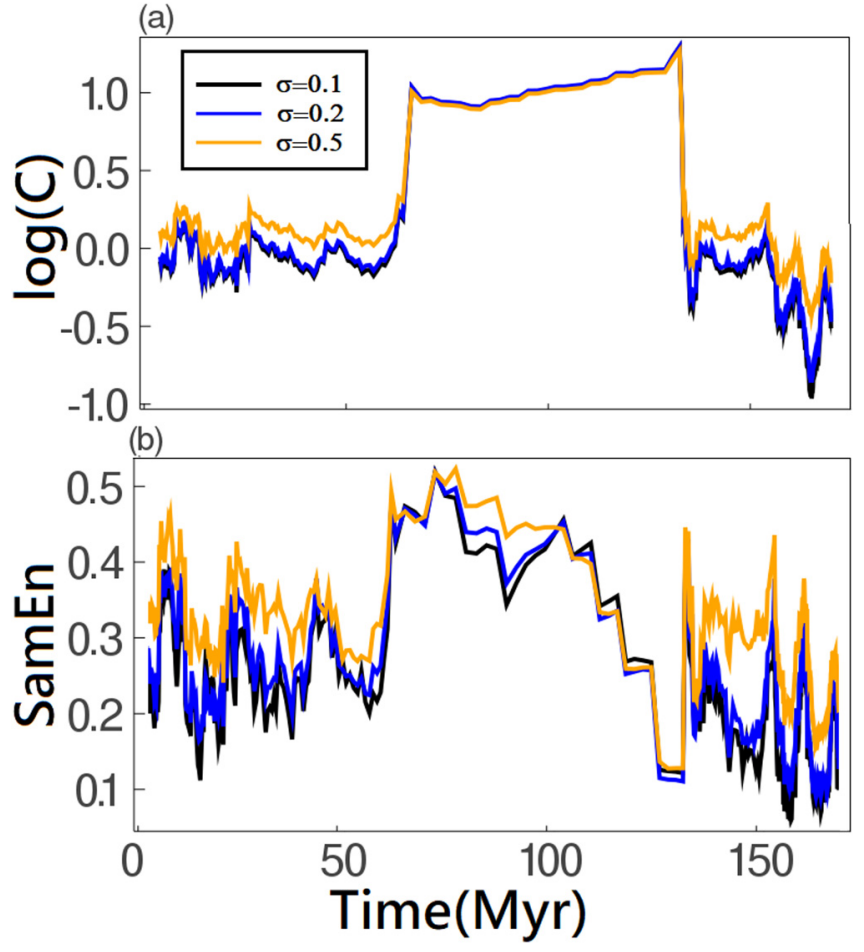

FIG. 3. Robustness test of the (a) coefficient of variation $(C)$ and (b) sample entropy (SamEn) calculated for the geomagnetic polarity scale using parameters $r=0.2 \sigma(X)$ and $m=2$ presented as a function of the number of the interval of the polarity scale. We present the results for levels of noise $\sigma=0.1$ (red), $\sigma=0.2$ (blue), and $\sigma=0.5$ (orange). Results show the stability of our results even for large levels of noise $(\sigma=0.5)$. The test is performed by generating 1000 independent realizations of $\epsilon_{i}$ the curves in (a) and (b) result from averaging 1000 randomly perturbed curves of $C$ and SamEn.

This measure is very robust to the influence of the noise, even with very strong variance ( $\sigma=0.5$ ) our conclusions still remain valid. The same test was performed for the SamEn with similar results. Since $C$ and SamEn are measures of organization their values increase as data become less predictable due to random errors. Consequently, the introduction of random errors introduced in the interval duration cannot reduce the measures $C$ and SamEn, but only increase them. This fact gives us an indication that the decreased values in $C$ and SamEn observed in the pre-154.3 Myrs era should not be a mere artifact of the inadequacies of the data set unless systematic/nonrandom errors have been introduced in the polarity timescale for some reason.

Also, in face of possible uncertainties arising in the pre154.3 Myrs data, we focus only on particular events of high regularity, measured by the coefficient of variation, in the last 154.3 Myrs. To implement this procedure, we calculate the coefficient of variation for a shorter sliding window corresponding to 15 consecutive intervals. Since these events are associated with fast reversal rates, we choose to plot the data replacing the $x$ axis from time (in Myrs) to the number of the polarity interval in the geomagnetic polarity timescale. With this, the events of organized reversals that would be otherwise squeezed in the $x$ direction become more evident. We present such analysis in Fig. 4. In this figure we identify four events of

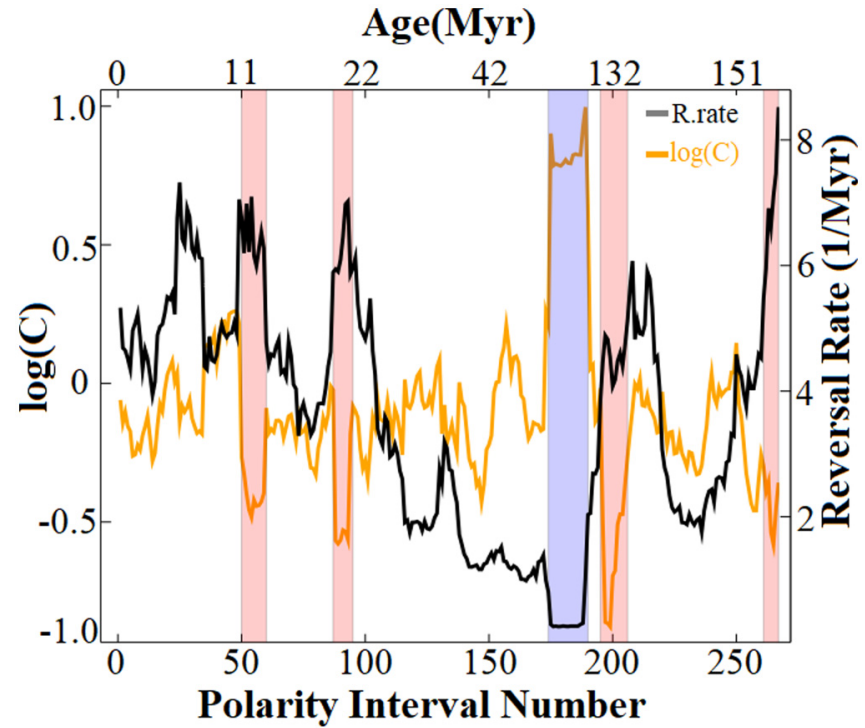

FIG. 4. Reversal rate (black curve) and the logarithm of the coefficient of variation (orange curve) as a function of the polarity interval number. The epochs with organized reversals are signaled with a red background in contrast with the disordered reversal sequences in the vicinity of the Cretaceous Normal Superchron marked with a blue background.

organized reversals in the last 154.3 Myrs. The first one refers to the event around $152 \mathrm{Myrs}$, the second one occurs around 130 Myrs ago preceding the Cretaceous Normal Superchron by about 10 million years. In more recent times two events of organized reversals were identified at around 20 and 12 Myrs ago. All of these events are accompanied by a substantial increase in the reversal rates and are marked with a red background in Fig. 4. On the other hand, the sequences containing the Cretaceous Superchron are highly disordered, this period is highlighted with a blue background in this figure. Here the inverse relationship between the coefficient of variation and the reversal rates becomes even more evident. This also highlights the fact that events of organized reversals are relevant even if we exclude the extreme reversal rates of the mid-Jurassic that can be seen in Fig. 2 .

Besides, to support the occurrence of the periods with organized reversals across the most reliable part of the data set, i.e., in the last 154.3 Myrs, in Fig. 5 we point out the prevalence of events of high reversal rates of $\approx 70$ kyrs. Moreover, the multipeak profile of the histogram presented in Fig. 5 is interpreted in the context of nonlinear dynamics as signatures of systems approaching bifurcations leading to periodic behavior. Hence, such a signature of regularity remains relevant even if the system is in a chaotic regime.

\section{DYNAMICAL SYSTEM ANALYSIS}

The use of simplified systems is of great value for the interpretation of transitions in natural systems. For instance, in hydrodynamical systems important dynamical features of low dimensional models are present despite the actual system has infinite degrees of freedom [34,35]. This is usually due to the predominance of a few modes in a suitable spectral 


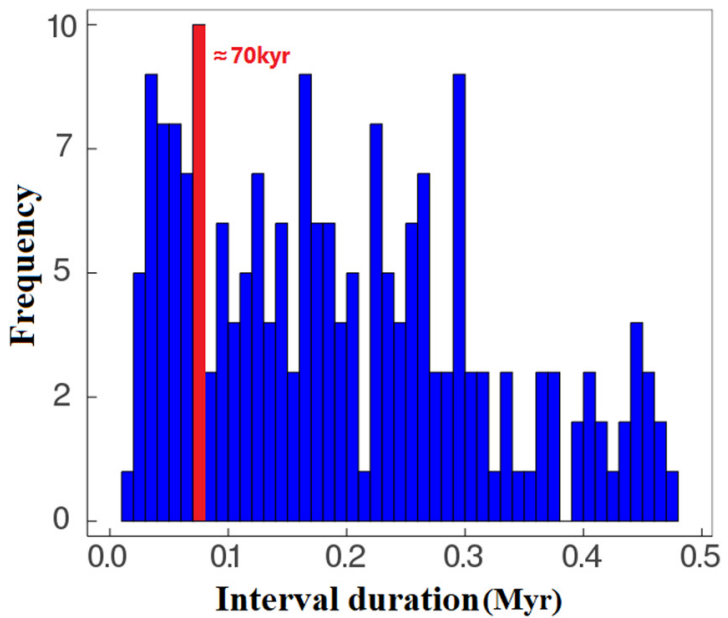

FIG. 5. Histogram of geomagnetic interval duration for the last 154.3 Myrs. This is obtained by truncating for events with a duration of $\leqslant 500$ kyrs.

decomposition. Estimates of the dimension of the reconstructed attractor associated with the geomagnetic axial dipole using embedding techniques on paleointensity data [36] indicate its dynamics is indeed low dimensional (3-7 dimensions). There are several simplified systems describing the dynamics of geomagnetic reversals, including stochastic systems in a bistable potential [32,33], stochastic systems with periodic forcings leading to the stochastic resonance phenomena $[30,37]$. However, we argue that a system with transitions driven purely by noise may not be able to capture some changes of behavior in the geomagnetic reversals such as transitions to superchrons [38] and possibly more regular geomagnetic reversals states as described in the previous section. As in [38] we share the view that the machinery of bifurcation theory might be necessary to describe these changes of behavior.

We use a reduced model for geomagnetic reversals introduced by Gissinger in Ref. [39] and further analyzed in Ref. [40]. It consists of a truncation of the magnetohydrodynamic equations that are consistent with the symmetry properties of the system. It retains only the dipole and quadruple components of the magnetic field, respectively, $D$ and $Q$, coupled with a symmetry-breaking flow component $V$. Despite the simplicity of the model, it presents a significant resemblance with the qualitative behavior of the geodynamo and a surprisingly rich variety of regimes, including periodic windows, superchronlike nonreversing states, and irregular/chaotic reversal regimes. The ability of this simple system in describing different behaviors of the geomagnetic dipole is robust. Specially because it consists of a normal form truncation respecting the symmetries of the MHD equations, therefore, the nature of the bifurcations found in this model are also expected to occur on the geodynamo system. In addition, Gissinger's model was used to perform data assimilation with coarse observations in order to estimate the expected time for the next dipole's reversal [41]. Compared to other simplifications, Gissinger's model had the best performance for this task. Furthermore, we argue that stochastic models in bistable potentials commonly used to represent geomagnetic reversals possess smooth exit-time probability distribution function (PDFs) [42], even in the presence of periodic forcing/stochastic resonance [43]. Therefore, this type of stochastic models are hard to relate to the type of distributions with a seemingly singular phenomenon portrayed in Fig. 2 . Furthermore, no second harmonic of the $\approx 70$ kyrs peak is found in the distribution observed in Fig. 2 suggesting that it is not due to stochastic resonance. Here we argue that the singular peak $\approx 70$ kyrs combined with the regular-irregular transitions are reminiscent of bifurcations leading to chaos in deterministic dynamical systems. Finally, the other (more smooth) peaks observed in Fig. 2 can be related to perioddoubling bifurcations in deterministic dynamical systems.

The low dimensional model is given by

$$
\begin{aligned}
& \dot{Q}=\mu Q-V D, \\
& \dot{D}=-\nu D+V Q, \\
& \dot{V}=\Gamma-V+Q D .
\end{aligned}
$$

The parameters $\mu, \Gamma$ can be seen as forcing terms on the quadrupole $Q$ and velocity field $V$ components, while the parameter $v$ can be seen as an effective viscosity term leading to the dissipation of the dipole component $D$ of the geomagnetic field. By performing a bifurcation analysis of this model, Gissinger has already demonstrated the existence of intervals in the parameter $\mu$ for which the system exhibit stable periodic behavior, i.e., periodic windows (PWs) [40]. Here we associate the high levels of regularity observed in Fig. 2 to the occurrence of PWs in the model. We argue that more than just the stable periodic behavior within every PW, the chaotic behavior of parameter regions adjacent to them contains vestiges of their periodicity.

Before we demonstrate the occurrence of PWs in the model describing the polarity of the geomagnetic field, we first emphasize that their occurrence is ubiquitous across a large variety of systems. In fact, their existence has been verified in many mathematical models [44] and in independent laboratory experiments $[45,46]$. In addition, such PWs have been found to occur with many different internal periods and in many scales of dynamical systems parameter space [47-49]. With this, for the model defined in Eq. (3) with effective viscosity at $v=0.1$, we obtain a two-dimensional stability diagram by computing the system's largest Lyapunov exponent $\left(\lambda_{\max }\right)$ [50] as a function of the parameters $\mu$ and $\Gamma$. Hence, in Fig. 6 we show such a stability diagram with the parameters leading chaotic solutions $\left(\lambda_{\max }>0\right)$ denoted in blue and yellow, while the PWs, i.e., the parameters leading to regular behavior $\left(\lambda_{\max }<0\right)$, are indicated in grayscale. By inspecting this diagram, we point out the occurrence of PWs of different sizes intermingled with parameters leading to chaos. We remark that the PWs are also present in smaller scales of the obtained diagram that cannot be resolved in Fig. 6.

In order to illustrate these ideas, in Fig. 7(a) we perform a bifurcation analysis of the system in a Poincaré section defined as $\Sigma=\left\{(Q, D, V) \in \mathcal{R}^{3} \mid a Q+b D=0\right\}$ with $a=\sqrt{\mu+\Gamma \sqrt{\mu / v}}$ and $b=\sqrt{v+\Gamma \sqrt{v / \mu}}$. In Fig. 7(a), for $\mu \in[0.113,0.118]$ (dashed line in Fig. 6), we observe many PWs in a sequence, it is worth noticing that even when the periodic orbits lose stability, become unstable periodic orbits (UPOs), and lead to chaotic behavior, the density of points 


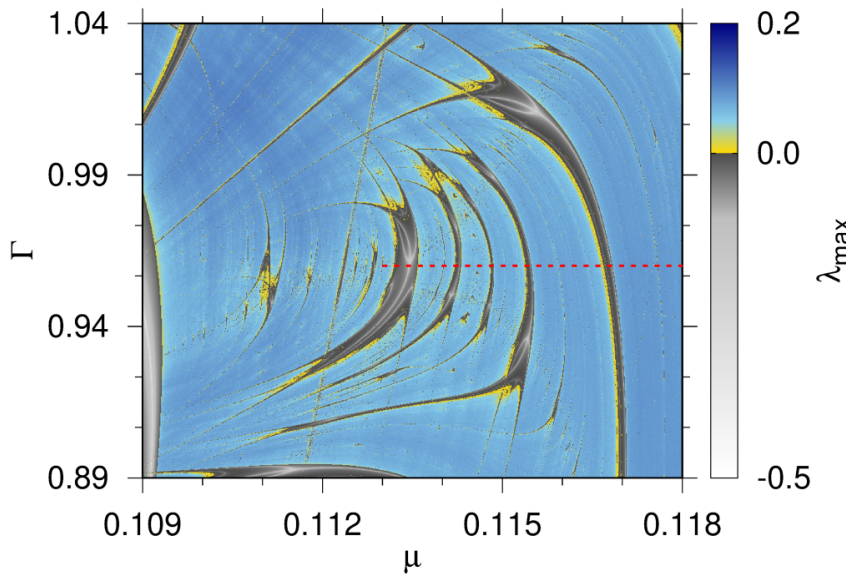

FIG. 6. Two-dimensional parameter space of the Gissinger's model defined in Eq. (8). The color code stands for the value of the largest Lyapunov exponent $\lambda_{\max }$. Blue and yellow indicate parameters leading to chaotic behavior $\left(\lambda_{\max }>0\right)$, while grayscale stands for the PWs, i.e., regular behavior $\left(\lambda_{\max }<0\right)$. The effective viscosity is at $v=0.1$. The dashed line indicates the parameters used in the subsequent analysis.

continue to be higher near the dominant UPOs. In Fig. 7(b), for the same interval of $\mu$, we show the maximum Lyapunov exponents $\left(\lambda_{\max }\right)$, the PW corresponds to $\lambda_{\max }<0$. Next, in Fig. 7(c) we obtain the sample entropy (SamEn) and the variation coefficient $(C)$ in the fashion of Fig. 2. Both SamEn and $C$ efficiently capture the occurrence of PWs in the model,

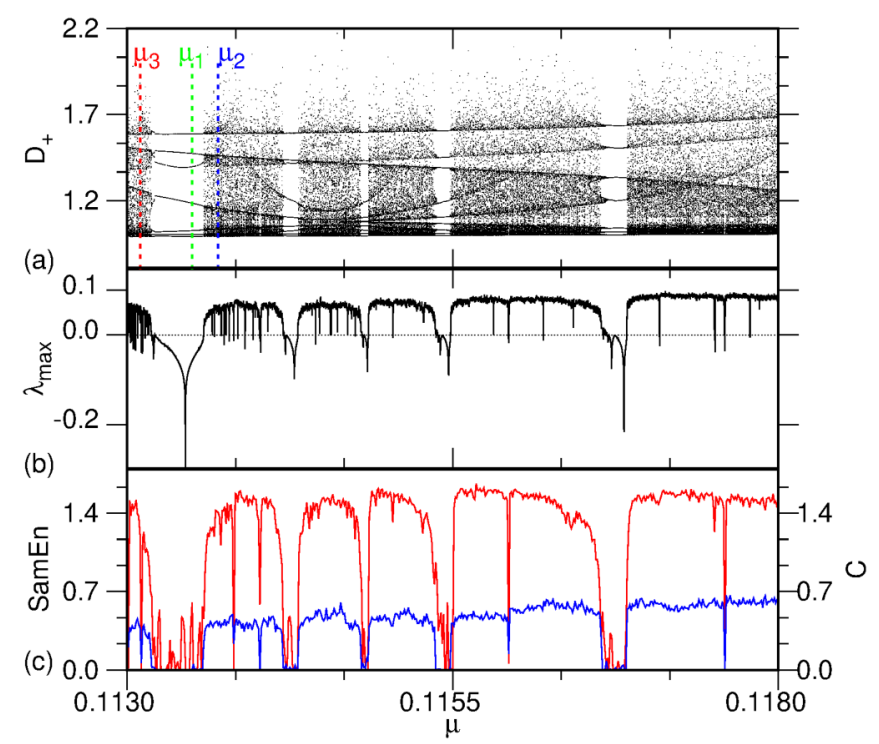

FIG. 7. (a) Bifurcation diagram of the Gissinger's model revealing parameter intervals leading to periodic behavior (PWs) and intervals leading to chaos. The bifurcation parameter $\mu$ is considered in the interval $[0.1130,0.1180]$. The green dashed line corresponds to $\mu_{1}=0.1135$, the blue dashed line indicates $\mu_{2}=0.1137$, and the red line $\mu_{3}=0.1131$. (b) The corresponding maximum Lyapunov exponents $\lambda_{\max }$. (c) Blue represents coefficient of variation $C$ and red stands for the sample entropy SamEn. The other system parameters are fixed at $v=0.1$ and $\Gamma=0.96$. supporting the capability of these measures in detecting the regularities of reversals data reported in Fig. 2.

In more detail, every $\mathrm{PW}$ in the bifurcation diagram shown in Fig. 7(a) is delimited by a saddle-node bifurcation on its right side and a period-doubling route to chaos on its left side. In the chaotic regime occurring in the neighborhood of the saddle-node bifurcation (right side), a Pomeau-Manneville type-I intermittency scenario [51,52] gives rise to ghost limit cycles (GLCs) [53,54]. An intermittent transient periodic behavior with the same periodicity of the main orbit within the adjacent PW. On the PW's left side, the periodic orbits created in the PW continue to exist in an unstable way, as an UPO [55]. Hence, for values of $\mu$ close enough to PWs on both sides, regularity will appear in the frequency of reversals.

UPOs can be viewed as the skeleton of a chaotic attractor, so that statistical properties of the system can be interpreted in terms of the properties of the UPOs [56,57]. The influence of UPOs has been previously discussed in the context of reversal rates of the geomagnetic field in [58]. In this study, the authors show that the invariant probability distribution of the system can be well approximated by the dominant UPOs of the system. These orbits can be classified into two types: the global UPOs are the ones that perform a reversal (crossing the plane $D=0$ ), and the local ones, which are responsible for variations of the geomagnetic field without reversing in the polarity polarity. In particular, during transitions to geomagnetic superchrons, it is suggested that the set of global UPOs is destroyed. Since UPOs are saddle orbits, their stable manifold attracts the chaotic orbits that latter get expelled through the unstable one. For orbits sufficiently close to the stable manifold, the motion mimics the UPO for extended times leaving a statistical imprint in the distribution function on the phase space. These almost periodic transients can latter be used to trace an initial approximation of the UPO that can be refined by iterative techniques involving the monodromy matrix.

To better clarify the occurrence of different degrees of regularity in the model, in Fig. 8 we explore in detail its dynamical behavior for the three values of $\mu$ shown in Fig. 7(a). First, for $\mu_{1}=0.1135$, the system oscillates in a stable periodic orbit within the PW. Hence, in Fig. 8(a), we show a state-space projection $(Q \times D)$ of such periodic oscillation (limit cycle) and in Fig. 8(d) we show the respective time evolution of the dipole component oscillating regularly. Next, for $\mu_{2}=0.1137$, the system oscillates chaotically, yet, under the effect of the ghost limit cycle (GLC). Hence, in Fig. 8(b), in red, we show a state-space projection of the underlying chaotic attractor and in black we show the intermittent transient periodic behavior corresponding to the GLC. In addition, the similarity between the stable limit cycle and its ghost can also be seen in some phases of the time evolution shown in Fig. 8(e). Finally, for $\mu_{3}=0.1131$, the reversal dynamics is also chaotic, however, it occurs in a parameter region where the periodic orbit of the neighbor PW exists in an unstable way, an UPO. In Fig. 8(c) we show in black an approximation of this dominant UPO overlapping the chaotic attractor shown in red. The time evolution of the dipole component $D$, shown in Fig. 8(f), further illustrates the intermittent transient regular behavior of the oscillation close to the UPO. The similarities 

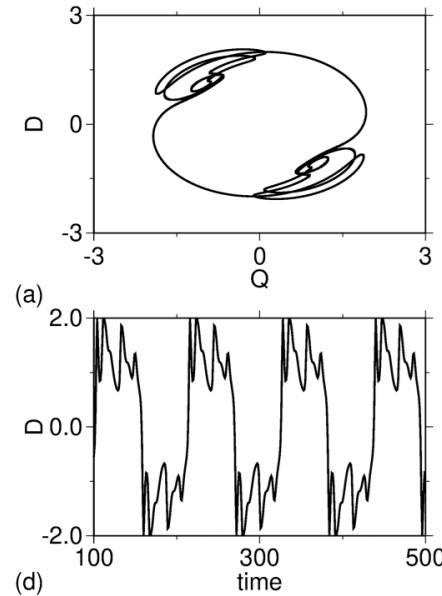

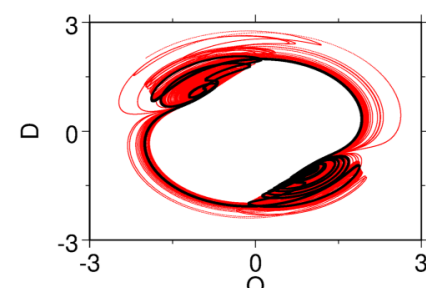

(b)

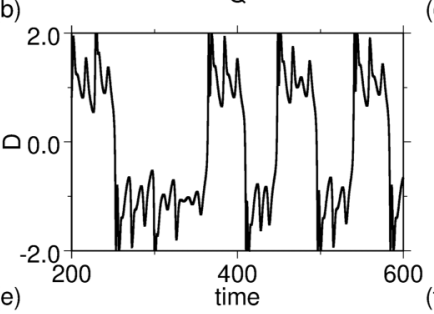

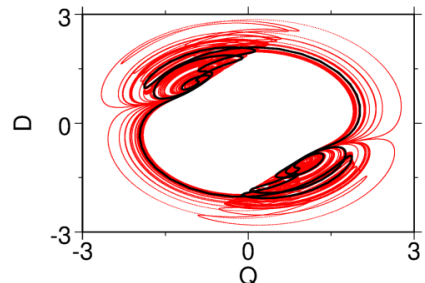

(c)

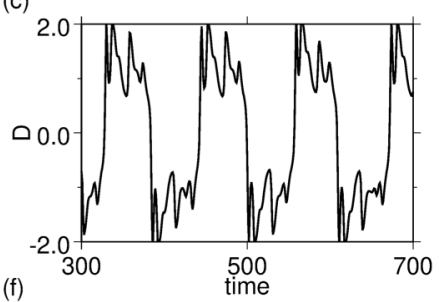

FIG. 8. (Top) State-space projection of the quadruple and dipole $(Q \times D)$ polarity components of the Gissinger's model. (a) For $\mu_{1}=$ 0.1135 , the system oscillates periodically in a limit cycle shown in red. (b) For $\mu_{2}=0.1137$, the trajectory follows a chaotic attractor shown in red. The intermittent transient behavior induced by the GLC is shown in black. (c) For $\mu_{3}=0.1131$, the system is also chaotic. The correspondent chaotic attractor is shown in red, while the dominant UPO is shown in black. (Bottom) Time evolution of the model's dipole component. (d)-(f) For the parameters corresponding to (a)-(c). The other system parameters are fixed at $v=0.1$ and $\Gamma=0.96$.

between the trajectories denoted in black in Fig. 8 express the underlying periodicities occurring in the model.

To further illustrate the impact of GLCs and UPOs in the long-term reversal dynamics of the model, we obtain the distribution of time intervals $\Delta T$ of the model's dipole component $D$ permanency in each field polarity. First, for the model following the limit cycle $\left(\mu_{1}=0.1135\right)$, in Fig. 9(a) we show the respective distribution of time intervals $\Delta T$ for each polarity of the model's dipole component $D$. The sole peak confirms the periodicity of the stable limit cycle, i.e., $\Delta T \approx 56$. Now, for $\mu_{1}=0.1137$, the model behaves chaotically, in the presence of the GLC. Hence, in Fig. 9(b) we show the corresponding distribution of polarity intervals $\Delta T$ for this parameter region. The high frequency of the polarity interval $\Delta T \approx 56$ indicates the high influence of the limit cycle nearby, i.e., its ghost. Next, for $\mu_{1}=0.1131$, the model also behaves chaotically, but in the presence of UPOs. With this, the most frequent polarity interval shown in Fig. 9(c) $\Delta T \approx 56$ indicates the dominance of the UPO associated with the PW's main periodic orbit. In addition, the presence of multiple peaks in the diagram shown in Fig. 9(c) indicates the occurrence of less dominant UPOs which could be associated with the multipeak structure presented in Fig. 2(c).

\section{DISCUSSIONS}

Based on measures of dispersion of sequences of consecutive polarity intervals, we have demonstrated the existence of signatures of regularity in the sequences of geomagnetic reversals in the last 170 million years, this includes:

(i) The detection of periods of more organized reversal sequences in the mid-Jurassic (160-150 Myrs) measured by sudden decreasing in the regularity measures, i.e., the coefficient of variation $(C)$ and the sample entropy (SamEn). A sequence of more regular reversals is also seen at the early Cretaceous $(\approx 130$ Myrs) preceding the Cretaceous Normal Superchron. Other events detected by the implemented measures occurs twice in the last 20 Myrs, one around 20
Myrs ago and the other around 12 Myrs ago. The degree of regularity of the sequences of geomagnetic reversals has an inverse relationship with the reversal rate. Every reduction in the measures of the regularity of subsequence of geomagnetic reversals corresponds to an increased reversal rate. On the other hand, periods of very low reversal rates, such as in the vicinity of the superchron, correspond to higher values of these measures.

(ii) The presence of a predominant fast-reversing rate period of $\approx 70$ kyrs corresponding to the highest peak in the histogram. This predominance is seen even if we exclude the fastest reversing period pre-154.3 Myrs (see Figs. 2 and 5).

(iii) The multipeak aspect of the histogram of geomagnetic polarity interval durations.

The existence of periods with high reversal rates in the geodynamo has a very significant impact on the statistics of chron duration [see histogram in Figs. 2(c) and 5]. A fast reversing rate period of $\approx 70 \mathrm{kyrs}$ corresponds to the largest peak in the histogram. While the clustering pattern in the statistics of intervals was already observed by [11] and may suggest the existence of different regimes in the geodynamo. This fast reversing and regular state are therefore highly relevant for the statistics of reversals.

The analogy with a simple model for the geomagnetic reversal [39] shows that bifurcations of the system may lead to periodic windows in which the chaotic attractor collapses to a limit cycle. This is caused by either a period-doubling route or by a type-I Pomeu Manneville intermittency. We show that even in the chaotic regime when the parameters are close to a periodic window, the limit-cycle ghost (GLCs) still impacts the dynamics leading to a large peak in the histogram of residence times, see Fig. 2(c). This suggests that even when the geodynamo operates in a chaotic regime the effects of GLCs, or UPOs can be felt, given that the system is close enough to a periodic window. Therefore, the large peak of $\approx 70$ kyrs in Fig. 2(c) could either be the signature of a limit cycle itself, its ghost, or its correspondent UPO. Furthermore, we emphasize that it may be difficult to directly observe the 

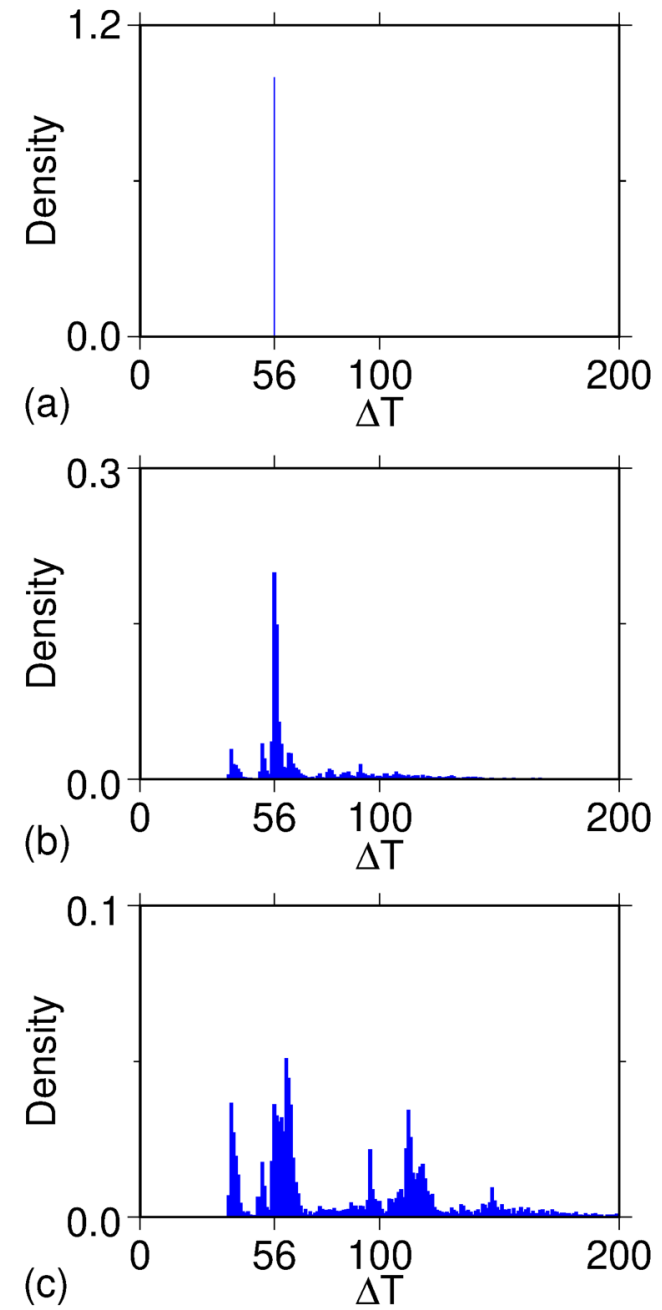

FIG. 9. The distribution of polarity intervals $\Delta T$ of the Gissinger's model [Eq. (3)]. (a) For $\mu_{1}=0.1135$, the system oscillates periodically in a limit cycle. (b) For $\mu_{2}=0.1137$, the model oscillates chaotically close to a GLC. (c) For $\mu_{3}=0.1131$, the system is also chaotic, but an UPO dominates the dynamics. The other system parameters are fixed at $v=0.1$ and $\Gamma=0.96$.

underlying stable periodic behavior from the polarity records, specially due to the system's parameters drifting through such smaller PWs. Nevertheless, we have demonstrated that overall effects of such stable periodicities can still be assessed.

The mechanism behind the variability of the duration of geomagnetic chrons is still elusive and it is, to a large extent, an open problem. Numerical geodynamo models suggest that the frequency of reversals depends crucially on the properties of the core-mantle boundary (CMB), in particular the heat flow in this interface [3]. Additionally, the geometry of the distribution of heat flux in the core-mantle boundary may be of high importance for the geodynamo, with evidence showing correlations with the tectonic process [59]. An inverse relationship between the degree of dipolarity of the geomagnetic field and the reversal rates was found in [60] which also coincides with the estimated heat flux at the CMB. Long term changes in this pattern may, therefore, alter the regime of operation of the dynamo.

It is also important to determine whether the peak at the histogram with $\approx 70$ kyrs has some relation with external forcing or if it results solely from the nonlinear variability of the system. Consolini and De Michelis [30] suggested that the 100 kyrs Millankovich orbital cycles could play a role in determining the frequency of reversals. In addition, they argued that stochastic resonance could also play a role and suggested that the distribution of polarity intervals is described as a superposition of Gaussians. Each one centered at multiples of a fundamental frequency $T=100 \mathrm{kyrs}$. This frequency has also been reported in the literature in inclination and intensity data [61].

The hypothesis of the Earth's orbital dynamics acting as one of the forcing mechanisms to the long-term geomagnetic field behavior remains a contentious subject in literature [61]. Some studies (e.g., [62]) advocated that the precessionally driven energy supply would not be significant to empower the outer core/mantle relative motion. However, it has been questioned more recently by other authors (e.g., [63]), indicating that the effect of orbital forcing on the geodynamo's energy budget cannot be ruled out. In the hypothesis of an orbitally driven origin of the $70 \mathrm{kyrs}$ signal, it cannot be straightforwardly attributed to one of the Earth's orbital parameters. Nevertheless, a similar multimillennial-scale quasiperiodicity has been reported as possibly resulted from distinctive processes-for instance, as a transient frequency associated with the eccentricity-precession modulation [64], as well as a short-eccentricity cycle expression during the minimal amplitude of the long-eccentricity cycle [65]. The lack of a clear evidence for a orbital forcing with period of $\approx 70 \mathrm{kyrs}$, and absence of a peak in a multiple of the fundamental period suggest that it is probably not a result of stochastic resonance. However, whether other peaks in the histogram in Fig. 2 arise purely from the internal geodynamo dynamics or some selforganizing/resonance associated with external orbital forcing, as in [30], remains to be investigated in future observational, theoretical, and numerical studies.

\section{ACKNOWLEDGMENTS}

This work was supported by FAPESP (Processes: 2017/23417-5, 2018/03211-6, 2013/26598-0). E.S.M. acknowledges support by the Deutsche Forschungsgemeinschaft (DFG, German Research Foundation) - Projektnummer 163436311 - SFB 910.
[1] P. H. Roberts and G. A. Glatzmaier, Geodynamo theory and simulations, Rev. Mod. Phys. 72, 1081 (2000).

[2] J. A. Tarduno, R. D. Cottrell, and A. V. Smirnov, The paleomagnetism of single silicate crystals: Recording geomagnetic field strength during mixed polarity intervals, superchrons, and inner core growth, Rev. Geophys. 44, RG1002 (2006).
[3] H. Amit and P. Olson, Lower mantle superplume growth excites geomagnetic reversals, Earth Planet. Sci. Lett. 414, 68 (2015).

[4] J. Bloxham, Sensitivity of the geomagnetic axial dipole to thermal core-mantle interactions, Nature (London) 405, 63 (2000). 
[5] S. C. Cande and D. V. Kent, Revised calibration of the geomagnetic polarity timescale for the late cretaceous and cenozoic, J. Geophys. Res.: Solid Earth 100, 6093 (1995).

[6] F. M. Gradstein, J. G. Ogg, M. Schmitz, and G. Ogg, The Geologic Time Scale 2012 (Elsevier, Amsterdam, 2012).

[7] M. W. Hounslow, Geomagnetic reversal rates following palaeozoic superchrons have a fast restart mechanism, Nat. Commun. 7, 12507 (2016)

[8] A. Cox, Lengths of geomagnetic polarity intervals, J. Geophys. Res. 73, 3247 (1968).

[9] M. McElhinny and P. L. McFadden, The Magnetic Field of the Earth: Paleomagnetism, the Core, and the Deep Mantle (Academic, New York, 1998), Vol. 63.

[10] C. Constable, On rates of occurrence of geomagnetic reversals, Phys. Earth Planet. Inter. 118, 181 (2000).

[11] V. Carbone, L. Sorriso-Valvo, A. Vecchio, F. Lepreti, P. Veltri, P. Harabaglia, and I. Guerra, Clustering of Polarity Reversals of the Geomagnetic Field, Phys. Rev. Lett. 96, 128501 (2006).

[12] R. Knaack and J. O. Stenflo, Spherical harmonic decomposition of solar magnetic fields, Astron. Astrophys. 438, 349 (2005).

[13] B. Raphaldini, E. Medeiros, C. F. Raupp, and A. S. Teruya, A new mechanism for Maunder-like solar minima: Phase synchronization dynamics in a simple nonlinear oscillator of magnetohydrodynamic Rossby waves, Astrophys. J. Lett. 890, L13 (2020).

[14] B. Raphaldini, A. S. Teruya, C. F. Raupp, and M. D. Bustamante, Nonlinear Rossby wave-wave and wave-mean flow theory for long-term solar cycle modulations, Astrophys. J. 887, 1 (2019).

[15] I. G. Usoskin, A history of solar activity over millennia, Living Rev. Sol. Phys. 14, 3 (2017).

[16] D. Ruelle, Chaotic Evolution and Strange Attractors (Cambridge University Press, Cambridge, 1989), Vol. 1.

[17] J.-P. Eckmann and D. Ruelle, Ergodic theory of chaos and strange attractors, in The Theory of Chaotic Attractors (Springer, Berlin, 1985), pp. 273-312.

[18] M. Viana and K. Oliveira, Foundations of Ergodic Theory (Cambridge University Press, Cambridge, 2016), Vol. 151.

[19] S. M. Pincus, Approximate entropy as a measure of system complexity, Proc. Natl. Acad. Sci. 88, 2297 (1991).

[20] J. S. Richman and J. R. Moorman, Physiological time-series analysis using approximate entropy and sample entropy, Am. J. Physiol.: Heart Circ. Physiol. 278, H2039 (2000).

[21] S. M. Pincus, I. M. Gladstone, and R. A. Ehrenkranz, A regularity statistic for medical data analysis, J. Clin. Monit. 7, 335 (1991).

[22] A. Porta, V. Bari, B. De Maria, B. Cairo, E. Vaini, M. Malacarne, M. Pagani, and D. Lucini, On the relevance of computing a local version of sample entropy in cardiovascular control analysis, IEEE Trans. Biomed. Eng. 66, 623 (2018).

[23] A. Delgado-Bonal and A. Marshak, Approximate entropy and sample entropy: A comprehensive tutorial, Entropy 21, 541 (2019).

[24] See Supplemental Material at http://link.aps.org/supplemental/ 10.1103/PhysRevResearch.3.013158 for additional sensitivity tests of the SamEn analysis for different parameters.

[25] M. D. Opdyke and J. E. Channell, Magnetic Stratigraphy (Academic, New York, 1996).
[26] C. G. Langereis, W. Krijgsman, G. Muttoni, and M. Menning, Magnetostratigraphy—concepts, definitions, and applications, Newsl. Stratigr. 43, 207 (2010).

[27] M. Tominaga, W. W. Sager, M. A. Tivey, and S.-M. Lee, Deeptow magnetic anomaly study of the Pacific Jurassic Quiet Zone and implications for the geomagnetic polarity reversal timescale and geomagnetic field behavior, J. Geophys. Res. 113, B07110 (2008).

[28] R. D. Müller, M. Sdrolias, C. Gaina, and W. R. Roest, Age, spreading rates, and spreading asymmetry of the world's ocean crust, Geochem. Geophys. Geosyst. 9, Q04006 (2008).

[29] N. Zhang and S. Zhong, Heat fluxes at the Earth's surface and core-mantle boundary since Pangea formation and their implications for the geomagnetic superchrons, Earth Planet. Sci. Lett. 306, 205 (2011).

[30] G. Consolini and P. De Michelis, Stochastic Resonance in Geomagnetic Polarity Reversals, Phys. Rev. Lett. 90, 058501 (2003).

[31] Y. Gallet, V. Pavlov, and I. Korovnikov, Extreme geomagnetic reversal frequency during the middle Cambrian as revealed by the magnetostratigraphy of the Khorbusuonka section (northeastern Siberia), Earth Planet. Sci. Lett. 528, 115823 (2019).

[32] B. Buffett and W. Davis, A probabilistic assessment of the next geomagnetic reversal, Geophys. Res. Lett. 45, 1845 (2018).

[33] P. Hoyng and J. Duistermaat, Geomagnetic reversals and the stochastic exit problem, Europhys. Lett. 68, 177 (2004).

[34] D. Faranda, Y. Sato, B. Saint-Michel, C. Wiertel, V. Padilla, B. Dubrulle, and F. Daviaud, Stochastic Chaos in a Turbulent Swirling Flow, Phys. Rev. Lett. 119, 014502 (2017).

[35] R. Temam, Infinite-Dimensional Dynamical Systems in Mechanics and Physics (Springer Science \& Business Media, New York, 2012), Vol. 68.

[36] D. A. Ryan and G. R. Sarson, The geodynamo as a low-dimensional deterministic system at the edge of chaos, Europhys. Lett. 83, 49001 (2008).

[37] S. Lorito, D. Schmitt, G. Consolini, and P. De Michelis, Stochastic resonance in a bistable geodynamo model, Astron. Nachr.: Astron. Notes 326, 227 (2005).

[38] B. Raphaldini and C. F. M. Raupp, Nonlinear dynamics of magnetohydrodynamic Rossby waves and the cyclic nature of solar magnetic activity, Astrophys. J. 799, 78 (2015).

[39] C. Gissinger, E. Dormy, and S. Fauve, Morphology of field reversals in turbulent dynamos, Europhys. Lett. 90, 49001 (2010).

[40] C. Gissinger, A new deterministic model for chaotic reversals, Eur. Phys. J. B 85, 137 (2012).

[41] M. Morzfeld, A. Fournier, and G. Hulot, Coarse predictions of dipole reversals by low-dimensional modeling and data assimilation, Phys. Earth Planet. Inter. 262, 8 (2017).

[42] Z. Schuss, Theory and Applications of Stochastic Processes: An Analytical Approach (Springer Science \& Business Media, New York, 2009), Vol. 170.

[43] N. Berglund and B. Gentz, Noise-Induced Phenomena in SlowFast Dynamical Systems: A Sample-Paths Approach (Springer Science \& Business Media, New York, 2006).

[44] C. Bonatto and J. A. C. Gallas, Accumulation boundaries: Codimension-two accumulation of accumulations in phase diagrams of semiconductor lasers, electric circuits, atmospheric and chemical oscillators, Philos. Trans. R. Soc. London Sect. A 366, 505 (2008). 
[45] R. Stoop, P. Benner, and Y. Uwate, Real-World Existence and Origins of the Spiral Organization of Shrimp-Shaped Domains, Phys. Rev. Lett. 105, 074102 (2010).

[46] E. R. Viana, Jr., R. M. Rubinger, H. A. Albuquerque, A. G. de Oliveira, and G. M. Ribeiro, High-resolution parameter space of an experimental chaotic circuit, Chaos 20, 023110 (2010).

[47] J. A. C. Gallas, Structure of the Parameter Space of the Hénon Map, Phys. Rev. Lett. 70, 2714 (1993).

[48] E. N. Lorenz, Compound windows of the Hénon-map, Physica D 237, 1689 (2008).

[49] E. Medeiros, I. Caldas, and M. Baptista, Sensitive dependence on parameters of continuous-time nonlinear dynamical systems, Chaos, Solitons Fractals 99, 16 (2017).

[50] A. Wolf, J. B. Swift, H. L. Swinney, and J. A. Vastano, Determining Lyapunov exponents from a time series, Physica D 16, 285 (1985).

[51] J.-P. Eckmann, Roads to turbulence in dissipative dynamical systems, Rev. Mod. Phys. 53, 643 (1981).

[52] Y. Pomeau and P. Manneville, Intermittent transition to turbulence in dissipative dynamical systems, Commun. Math. Phys. 74, 189 (1980).

[53] D. Ruelle, Elements of Differentiable Dynamics and Bifurcation Theory (Elsevier, Amsterdam, 2014).

[54] E. S. Medeiros, I. L. Caldas, M. S. Baptista, and U. Feudel, Trapping phenomenon attenuates the consequences of tipping points for limit cycles, Sci. Rep. 7, 42351 (2017).

[55] C. Grebogi, E. Ott, and J. A. Yorke, Unstable periodic orbits and the dimensions of multifractal chaotic attractors, Phys. Rev. A 37, 1711 (1988).

[56] V. Lucarini and A. Gritsun, A new mathematical framework for atmospheric blocking events, Clim. Dyn. 54, 575 (2020).
[57] A. Gritsun and V. Lucarini, Fluctuations, response, and resonances in a simple atmospheric model, Physica D 349, 62 (2017).

[58] B. Raphaldini, D. Ciro, E. S. Medeiros, L. Massaroppe, and R. I. F. Trindade, Evidence for crisis-induced intermittency during geomagnetic superchron transitions, Phys. Rev. E 101, 022206 (2020).

[59] F. Pétrélis, J. Besse, and J.-P. Valet, Plate tectonics may control geomagnetic reversal frequency, Geophys. Res. Lett. 38, L19303 (2011).

[60] D. R. Franco, W. P. de Oliveira, F. B. V. de Freitas, D. Takahashi, C. F. da Ponte Neto, and I. M. C. Peixoto, Paleomagnetic evidence for inverse correspondence between the relative contribution of the axial dipole field and CMB heat flux for the past 270 Myr, Sci. Rep. 9, 1 (2019).

[61] T. Yamazaki and H. Oda, Orbital influence on Earth's magnetic field: 100,000-year periodicity in inclination, Science 295, 2435 (2002).

[62] M. G. Rochester, J. A. Jacobs, D. E. Smylie, and K. F. Chong, Can precession power the geodynamo?, Geophys. J. R. Astron. Soc. 43, 661 (1975).

[63] U. R. Christensen and A. Tilgner, Power requirement of the geodynamo from ohmic losses in numerical and laboratory dynamos, Nature (London) 429, 169 (2004).

[64] L. A. Hinnov, New perspectives on orbitally forced stratigraphy, Annu. Rev. Earth Planet Sci. 28, 419 (2000).

[65] M. Hennebert, Hunting for the 405-kyr eccentricity cycle phase at the Cretaceous-Paleogene boundary in the aiin settara section (kalaat senan, central tunisia), Carnets Géologie CG2012A05, 93 (2012) 